\title{
Study on the Relationship between Pore Structure and Uniaxial Compressive Strength of Cemented Paste Backfill by Using Air-Entraining Agent
}

\author{
Fengwen Zhao, ${ }^{1}$ Jianhua Hu $\mathbb{D}^{D},{ }^{1}$ Dongjie Yang $\mathbb{D}^{1},{ }^{1}$ Ye Kuang, ${ }^{1}$ Hongxing Xiao, ${ }^{2}$ \\ Minghua Zheng, ${ }^{2}$ and Xueliang Wang ${ }^{2}$ \\ ${ }^{1}$ School of Resources and Safety Engineering, Central South University, Changsha, Hunan 410081, China \\ ${ }^{2}$ Hubei Sanning Mining Co., Ltd., Yichang, Hubei 443100, China \\ Correspondence should be addressed to Dongjie Yang; yangdjxx@csu.edu.cn
}

Received 5 January 2021; Revised 31 March 2021; Accepted 21 April 2021; Published 3 May 2021

Academic Editor: Tingting Zhang

Copyright (C) 2021 Fengwen Zhao et al. This is an open access article distributed under the Creative Commons Attribution License, which permits unrestricted use, distribution, and reproduction in any medium, provided the original work is properly cited.

\begin{abstract}
To control pores in the backfill, the air-entraining agents (AEAs) are used as an admixture to realize the pore structure changes under artificial action and explore the effect of pore structure on strength. Two AEAs at different dosages were added to the backfill. The relationship was then analyzed between them from the macro- and mesoscopic aspects. The results indicate that AEA can regulate pore structure changes of AEACPB. With the increase in AEA content, the total pore volume of different pore sizes in AEACPB increases, in which the proportion of big and medium pore gradually increases while the proportion of small pore gradually decreases. The AEACPB's UCS is linearly negatively correlated with the porosity and pore percentage, which is the primary factor affecting the AEACPB of the pore structure. When the total pores' volume in the AEACPB is constant, the influence of different pore structures differs. A higher proportion of small pores leads to a linear increase in strength; a higher proportion of medium pores leads to a linear decrease in strength; and a higher proportion of big pores leads to an exponential decrease in strength. And the fractal dimension has a linear negative correlation with the UCS by fractal theory analysis.
\end{abstract}

\section{Introduction}

As an important indicator of mine filling, the backfill strength has multiple influencing factors. They can be divided into macro and micro aspects according to the perspective of analysis. In the macroscopic aspect, the composition of filling material had a significant effect on strength. The results report that macro factors such as cement-sand ratio, slurry concentration, solids content of tailings, and fine tailings have a significant impact on strength [1-3]. In the microscopic aspect, this study was performed on microscopic substance and reported that the sulfate, type, shape, and quantity of hydration products have an important influence on strength [4-7]. However, the pore structure is a major factor affecting the pore material's strength [8-10].

The strength is considerably influenced by pores, and the pore structure is changed to achieve the influence strength. It is very important that to achieve the change of pore structure through some methods and means for to study the effect of pore on strength. Dai et al. examined the relationships between pores structure and strength by changing the water-binder ratio of concrete and reported that as the water-binder ratio increased, the average pores size increased while the porosity decreased, and the strength first increased and then decreased, indicating that the pores structure was an important factor determining the concrete strength [11]. Huo et al. then analyzed the relationship between harmful pores having a size of $>0.2 \mu \mathrm{m}$ and concrete strength in different curing ages. They reported that, during the curing period of 7-28 days, pore structure and uniaxial compressive strength (UCS) considerably changed; moreover, the pore structure and strength change tended to stabilize during the curing period of 28-90 days. Thus, there is a certain relationship between pore structure characteristics and strength [12]. However, these methods will not 
only cause pore structure changes but also affect other factors, and air-entraining agent (AEA) is a type of admixture that can introduce a large number of bubbles into the backfill to generate pores, thus changing the pore structure in the backfill. Note that it can change the pore structure in the backfill under the condition that the ratio of other materials remains unchanged so as to achieve an individual change of pores in the same age and achieve the purpose of artificially controlling the pore structure. Studies report that adding AEA to the pore material can effectively change the pore structure and improve the material's performance [13-15]. Furthermore, we performed quantitative description of pore structure to analyze the effect of pore on strength. Moreover, the analysis methods of pore structure primarily include mercury injection test, nuclear magnetic resonance (NMR), and scanning electron microscopy (SEM). Jiang et al. measured the porosity and pores size distribution of concrete by mercury injection [16]. Zou et al. observed the evolution law of the concrete mesopore structure in the curing process using SEM [17]. Hu et al. used SEM to observe and analyze the hydration products and pore structure characteristics in the backfill $[18,19]$. And Liu et al. examined the distribution characteristics of various pore sizes using NMR spectroscopy [20]. All of the abovementioned techniques can be used to analyze pores' characteristics and achieve the acquisition of relevant characteristics of pore structure. And there are not many studies on quantitative analysis of backfill pores by using these techniques.

The amount of data and quantitative study of pore and strength are insufficient in this study. Tailings, tailings sludge, fly ash, and other raw materials were used to manufacture backfill samples, whereas AEAs (sodium abietate (SA) and triterpenoid saponins (SJ)) were used to regulate the backfill's pore structure. They were cured in a standard curing box $\left(20^{\circ} \mathrm{C}\right.$ and $99 \%$ humidity) for seven days and then tested by UCS, NMR, and SEM. By establishing the relationship model between the strength of the cemented paste backfill with an additional airentraining agent (AEACPB) and the pores' structure, the quantitative relationship research between pore structure and the strength is then realized. Thus, the relationship between strength of backfill and pores is comprehensively analyzed.

\section{Materials and Methods}

2.1. Materials. Tailings, fly ash, and tailings sludge were obtained from the Sanning Mine, Hubei, China. They were analyzed using a sieving method, laser particle size analyzer (Mastersizer 2000), and X-ray diffraction (XRD). Their physical properties (Table 1) and particle size distribution (Figure 1 and 2) were as follows. AEAs were then provided by a supplier based in Shandong province, and the physical properties of the AEAs are shown in Table 2. In this study, Portland cement was sourced from Changsha Xinxing Cement Factory; its physical properties are shown in Table 3. Ordinary tap water of Changsha was used.
TABLE 1: Physical properties of the materials.

\begin{tabular}{lccc}
\hline Class & Tailings & Tailing sludge & Fly ash \\
\hline Apparent density $\left(\mathrm{kg} / \mathrm{m}^{3}\right)$ & 2626 & 2653 & 1990 \\
Packing density $\left(\mathrm{kg} / \mathrm{m}^{3}\right)$ & 1464 & 923 & 650 \\
Surface moisture content (\%) & 0.120 & 0.974 & 0.049 \\
Hydroxyllapatite (\%) & 10.15 & 60.94 & - \\
Quartz (\%) & 6.91 & 2.24 & 61.55 \\
Hematite (\%) & 12.75 & - & 1.46 \\
Albite (\%) & - & 8.38 & 15.99 \\
Plagioclase (\%) & - & 11.42 & - \\
Muscovite (\%) & - & - & 20.99 \\
Illite (\%) & - & 9.76 & - \\
Dolomite (\%) & 69.65 & 6.30 & - \\
\hline
\end{tabular}

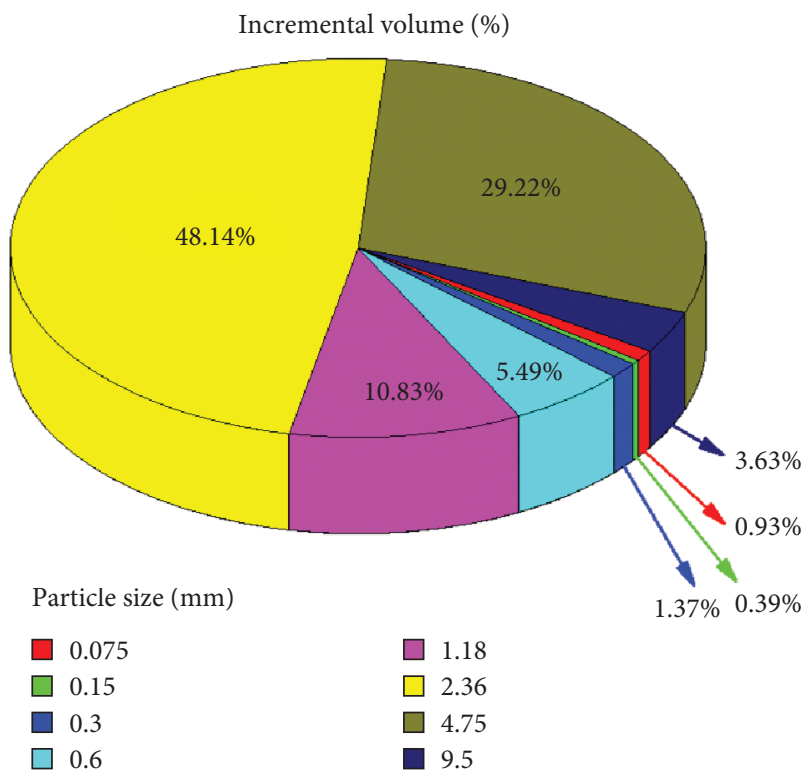

FIgure 1: Particle size distribution of tailings.

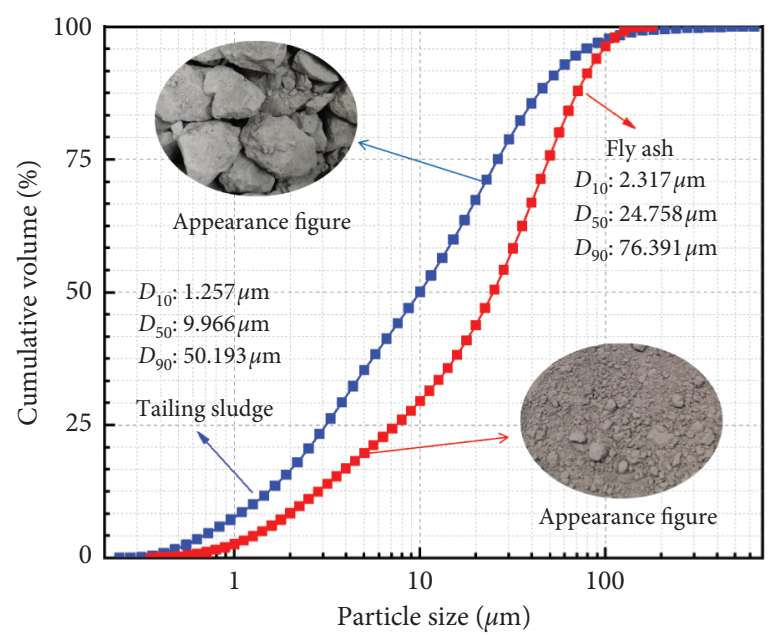

Figure 2: Particle size distribution of tailing sludge and fly ash.

\subsection{Experimental}

2.2.1. Experimental Scheme Design. Tailing and tailings sludge are regarded as aggregates. Cement and fly ash are 
TABLE 2: The main characteristics of used AEA.

\begin{tabular}{lcc}
\hline Main components & Appearance & $\mathrm{pH}$ \\
\hline SA & White powder & 10 \\
SJ & Pale-yellow powder & 6 \\
\hline
\end{tabular}

TABle 3: Physical parameters of the cement.

\begin{tabular}{lcccc}
\hline Chemical component & $3 \mathrm{CaO} \cdot \mathrm{SiO}_{2}$ & $2 \mathrm{CaO} \cdot \mathrm{SiO}_{2}$ & $3 \mathrm{CaO} \cdot \mathrm{Al}_{2} \mathrm{O}_{3}$ & $4 \mathrm{CaO} \cdot \mathrm{Al}_{2} \mathrm{O}_{3} \cdot \mathrm{Fe}_{2} \mathrm{O}_{3}$ \\
\hline Content & 52.8 & 20.7 & 11.5 & 8.8 \\
Physical properties & Apparent density $\left(\mathrm{kg} / \mathrm{m}^{3}\right)$ & Packing density $\left(\mathrm{kg} / \mathrm{m}^{3}\right)$ & - & - \\
Numerical value & 3100 & 1300 & - & - \\
\hline
\end{tabular}

regarded as cementing material. In this study, the dosages of AEAs (SA and SJ) include three types. In order to meet the requirement of the Tiaoshuihe Phosphate Mine, the ratio of cement to tailing is $1: 10$ and concentration is $80 \%$. The specific ratios (Table 4 ) of this experiment are as follows.

The manufacturing of AEACPB specimen according to GB/T50081-2002 is as follows. First, every raw material is weighed as per the design requirement, and these materials are mixed evenly by blender. Then, the filling slurry is injected into cylindrical mold $(\varphi 50 \times 100 \mathrm{~mm})$. And the specimens are cured in a curing box $\left(20^{\circ} \mathrm{C}\right.$ and $99 \%$ humidity) for 7 days after demolding. Finally, the samples were tested with SEM, NMR, and UCS.

\subsubsection{Experimental Test}

(i) SEM analysis is undertaken using a Quanta-200. The test objects are fragments of $1 \mathrm{~mm}^{2}$ picked from the center of damaged samples. Moreover, surface dusts are cleared and metal conductive films are applied before test.

(ii) NMR analysis is undertaken using a MesoMR23$060 \mathrm{H}$. The test objects are complete samples cured for 7 days. First, they are pumped dry and then soaked, so as to ensure they are fully saturated. Finally, the samples are tested.

(iii) The UCS test is performed using a compressor no. 01000405 , and its loading rate is $0.2 \mathrm{kN} / \mathrm{s}$. The UCS value is then obtained through a force measurement system analysis. The reference standard for this experiment is ASTM D2166/D2166M-16.

The experiment process is shown in Figure 3.

\section{Results}

\subsection{NMR Results}

3.1.1. $T_{2}$ Spectrum Distribution. For the AEACPB sample treated with saturated water, NMR $T_{2}$ spectrum can reflect the pore size distribution feature of the pores in the AEACPB. Furthermore, there is a certain functional relationship between the pore size and $T_{2}$, which can be expressed as follows [21, 22]:
TABLE 4: Mixture proportions for the AEACPB.

\begin{tabular}{|c|c|c|c|c|c|c|}
\hline \multirow[b]{2}{*}{ Code } & \multirow[b]{2}{*}{ Tailings } & \multirow[t]{2}{*}{$\begin{array}{l}\text { Tailing } \\
\text { sludge }\end{array}$} & Fly ash & Cement & & SA SJ \\
\hline & & & \multicolumn{2}{|l|}{$\left(\mathrm{kg} / \mathrm{m}^{3}\right)$} & Water & $\begin{array}{c}\text { By wt } \% \\
\text { of the } \\
\text { cement }\end{array}$ \\
\hline SA1 & 1500 & 400 & 200 & 150 & 562 & $0.2-$ \\
\hline SA2 & 1500 & 400 & 200 & 150 & 562 & 0.4 \\
\hline SA3 & 1500 & 400 & 200 & 150 & 562 & 0.6 \\
\hline SJ1 & 1500 & 400 & 200 & 150 & 562 & - \\
\hline SJ2 & 1500 & 400 & 200 & 150 & 562 & - \\
\hline SJ3 & 1500 & 400 & 200 & 150 & 562 & -0.6 \\
\hline
\end{tabular}

Note. "wt\%" represents solid mass percentage.

$$
r \approx T_{2} \rho_{2} F_{s},
$$

where $r$ is pore radius $(\mu \mathrm{m}) ; T_{2}$ is transverse relaxation time (ms); $\rho_{2}$ is transverse surface relaxation strength $(\mu \mathrm{m} / \mathrm{ms})$; and $F_{s}$ is pores' shape factor (for spherical pore, $F_{s}=3$, and for tube bundle pore, $F_{s}=2$ ).

Because pores' structure of AEACPB was similar to that of concrete, the parameter $\rho_{2}(12 \mathrm{~nm} / \mathrm{ms})$ could be got as per related study [23]. AEA could densify pore, so the pore could be regarded as spherical shape $\left(F_{s}=3\right)$. Therefore, the relationship between $r$ and $T_{2}$ is as follows:

$$
r \approx 36 \times 10^{-9} \times T_{2} \text {. }
$$

Combining equation (2) and $T_{2}$ spectrum, the $T_{2}$ spectrum included pore size and $T_{2}$ value can be got (Figure 4). Figure 4 shows that the $T_{2}$ spectrum is primarily divided into three peaks from big to small; moreover, it can then be divided into small, medium, and big pores, of which the small pores are the main ones (the largest area). As the AEA content increases, the first peak in the $T_{2}$ spectrum gradually increases, which shows that the small pores gradually increase with the increase of AEA content. However, the second and third peaks did not change much, indicating that the AEA had little effect on the medium and big pores. In Figure 4, $20 \mathrm{~nm}$ is the boundary of the pore size whether it is harmful to the strength. As per relevant research, when the pore size is $>20 \mathrm{~nm}$, the pores are harmful to the strength; otherwise, they are not harmful [24]. Figure 4 shows that the small pores in the first peak of the $T_{2}$ spectrum are primarily pores of $<20 \mathrm{~nm}$, which have little 


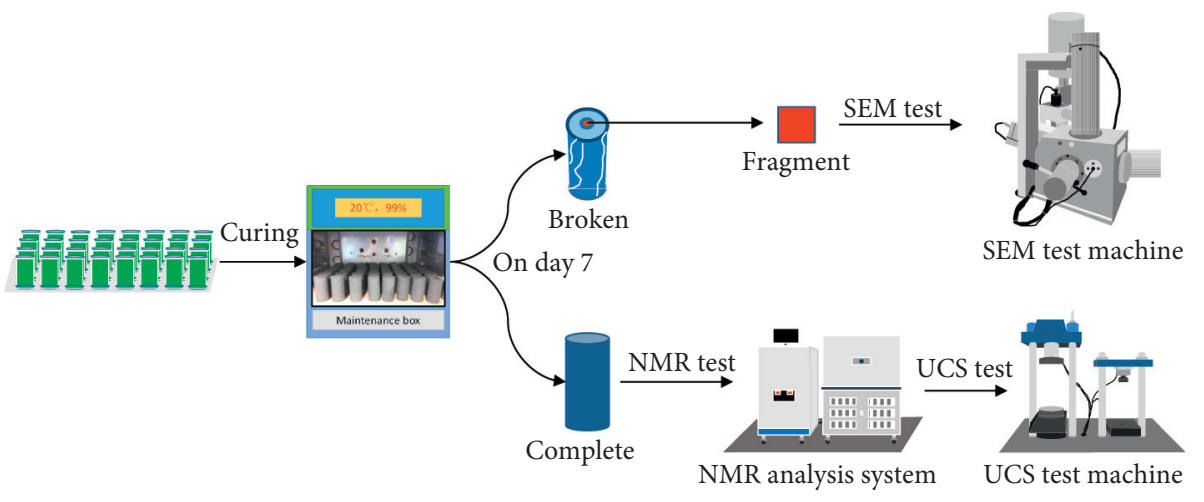

FIGURE 3: Experiment process with sample preparation, UCS tests, and SEM.

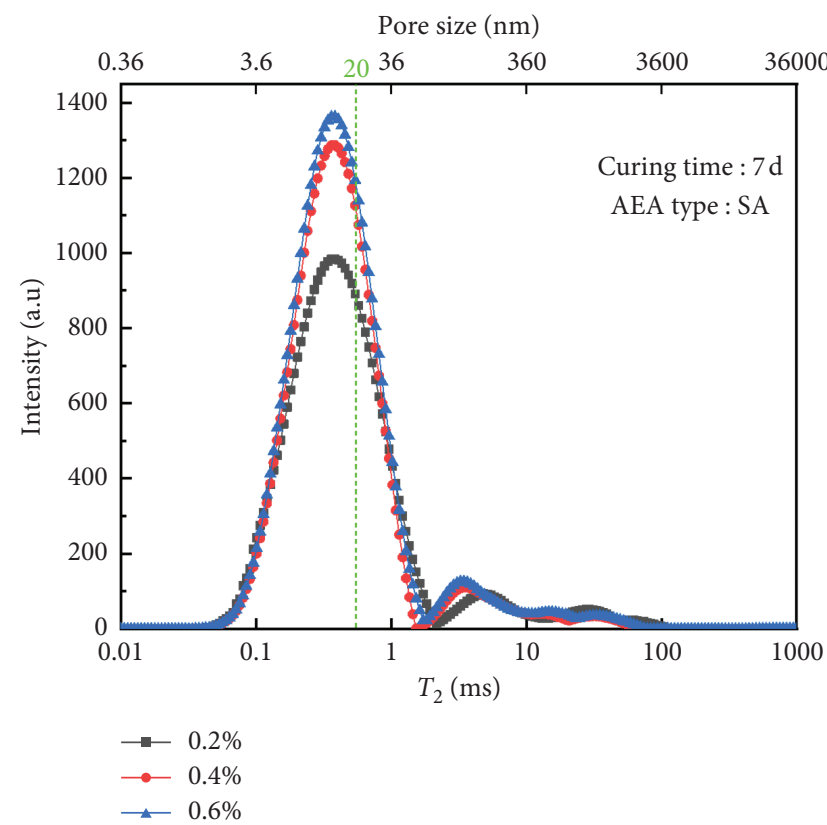

(a)

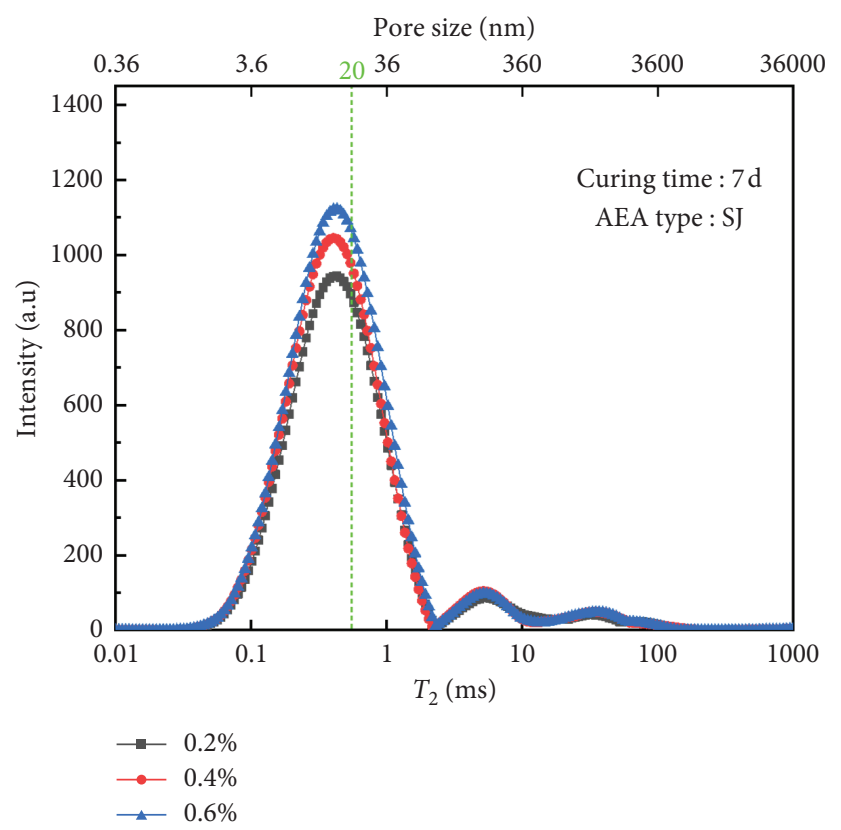

(b)

Figure 4: NMR $T_{2}$ spectral distribution of AEACPB samples incorporating different concentration of AEA.

impact on the strength deterioration. However, the second and third peaks are primarily medium and big pores of $>20 \mathrm{~nm}$, which have a harmful effect on strength.

3.1.2. $T_{2}$ Spectrum Peak Area. In the AEACPB sample, there is a certain correlation between the $T_{2}$ spectral area and the amount of fluid contained. The peak areas and their proportions reflect the number of pores and their proportions in the AEACPB sample. Table 5 shows the peak areas and their proportions in the AEACPB samples under different AEA contents. Table 5 shows that as the content of the AEA increases, both the area of each peak and the total area gradually increase, which indicates that the AEA addition introduces a large number of bubbles, which makes the pore volume increase. However, the proportion of the first peak area decreases, indicating that although a large number of bubbles can be introduced with increase in AEA content, the proportion of small bubbles decreases and the proportion of big and medium bubbles gradually increases, which is harmful to AEACPB strength.

3.2. SEM Analysis. In addition to using NMR, SEM can be used to study the distribution feature of pores in the AEACPB sample. The SEM images can be used to observe the pore distribution feature of some areas in the AEACPB sample. The SEM image is then processed using binarization, and the distribution of black and white areas in the binarization image can reflect pore distribution. The binarization processing is that the solid part and pore part in SEM image can be transformed into black and white regions by using threshold grayscale segmentation. The processing principle is as follows $[25,26]$ :

$$
f(i, j)= \begin{cases}0, & f(i, j)<T, \\ 1, & f(i, j) \geq T,\end{cases}
$$

where $T$ is the threshold grayscale. 
TABLE 5: $T_{2}$ spectral peak areas and their proportions of AEACPB samples doped with different AEA.

\begin{tabular}{|c|c|c|c|c|c|c|c|c|}
\hline \multirow{2}{*}{ AEA type } & \multirow{2}{*}{ Dosage (\%) } & \multicolumn{7}{|c|}{ Peak area and its proportion } \\
\hline & & The first & Proportion & The second & Proportion & The third & Proportion & Total area \\
\hline \multirow{3}{*}{ SA } & 0.2 & 32467.06 & 0.9167 & 1825.85 & 0.0516 & 1124.81 & 0.0318 & 35417.72 \\
\hline & 0.4 & 37077.48 & 0.9141 & 2113.89 & 0.0521 & 1368.23 & 0.0337 & 40559.60 \\
\hline & 0.6 & 40486.61 & 0.9094 & 2511.36 & 0.0564 & 1524.25 & 0.0342 & 44522.23 \\
\hline \multirow{3}{*}{ SJ } & 0.2 & 29724.96 & 0.9274 & 1407.53 & 0.0439 & 918.32 & 0.0287 & 32050.81 \\
\hline & 0.4 & 32293.20 & 0.9162 & 1803.33 & 0.0512 & 1150.57 & 0.0326 & 35247.10 \\
\hline & 0.6 & 36562.98 & 0.9126 & 2141.14 & 0.0534 & 1361.33 & 0.0340 & 40065.45 \\
\hline
\end{tabular}

According to equation (3), the SEM binarization image is composed by setting pixel point to 0 where the gray level is lower than $T$, i.e., the black point (solid part) and setting pixel point to 1 where the gray level is higher than or equal to $T$, i.e., the white point (pore part). The porosity that is measured is from the macro aspect using NMR, but SEM can be used to obtain the pore percentage from the micro aspect. The black and white areas in the binarization image are composed of a number of pixel points. The white areas in the binarization image are added, and the pore percentage of the sample can then be obtained by calculating the area percentage of the white area in the area of the entire picture. Figure 5 shows the SEM image and its binarization image of AEACPB samples. Figure 5 shows that the pores are primarily divided into three forms, namely, small, medium, and big pores. Among them, there are primarily small pores, and the proportion of big and medium pores is not much, which is consistent with the results obtained in Section 3.2.

3.3. Porosity and Pore Percentage. Porosity and pore percentage are two methods for calculating the total pore volume percentage of AEACPB. The porosity is directly obtained by NMR, but the pore percentage is obtained by obtaining the SEM binarization image. Figure 6 shows the changes of porosity and pore percentage with AEA content. Figure 6 shows that as the content of the AEA increases, both the porosity and the pore percentage increase; however, the pore percentage is lesser than the porosity. The total volume of the AEACPB sample remains unchanged; therefore, the amount of pores increased. This indicates that AEA can effectively increase amount of pore in AEACPB because AEA can introduce a large number of bubbles during the stirring process of filling slurry, and these bubbles will then form pores after the AEACPB is hardened. With additional AEA content, more bubbles will be introduced, resulting in an increase in the amount of the pore with an increase in AEA content. However, the main composition of SA-type and SJ-type AEA is different, and the bubbles produced by the two kinds of AEA are different. SA-type AEA will produce a large number of bubbles, while SJ-type AEA will produce a small number of bubbles, which leads to the difference of the backfill pore formed in the latter, and the porosity of the backfill with SA-type AEA is higher.

3.4. Strength Characteristics. Strength is an important feature of the $A E A C P B$, and its size is of great significance.
The strength change curve of the AEACPB was observed with the different contents of the two external AEAs on seven days (Figure 7). Figure 7 shows that as the content of the AEA increases, the strength gradually decreases, which indicates that AEA addition has a certain effect on the AEACPB strength. This is because the AEA can introduce a large number of bubbles in the AEACPB, resulting in a large number of pores in the AEACPB. Moreover, the different content of AEA can create pores with different pore sizes in the AEACPB, and pores with different pore sizes have different effects on the AEACPB strength. When the content of the AEA increases, pores with larger pore size will be generated in the AEACPB, which leads to a decrease in the AEACPB strength. It is shown in Section 3.3 that porosity increases with the increase of AEA content. The increase of porosity leads to the decrease of strength, and the porosity of the backfill with SA is greater than that of the backfill with SJ, so the strength of the backfill with SA is relatively low.

\section{Discussion and Analysis}

4.1. Relationship between Strength and Porosity and Pore Percentage. In the AEACPB sample, porosity embodies the total pore feature; moreover, it has an inevitable connection with strength. Certain studies have reported that there is a certain functional relationship between porosity and strength $[19,27]$. Now, two techniques are used to obtain the relationships between strength and porosity and pore percentage (Figure 8 ). Figure 8 shows that the relationship between porosity and strength is better $\left(R^{2}=0.92\right)$; however, that between pore percentage and strength is worse $\left(R^{2}=0.68\right)$. The relationships between them are as follows:

$$
\begin{aligned}
& y=-0.4 x_{1}+3.62, \\
& y=-0.31 x_{2}+2.84
\end{aligned}
$$

where $y$ is strength $(\mathrm{MPa}) ; x_{1}$ is porosity (\%); and $x_{2}$ is pore percentage (\%).

Figure 8 shows that no matter the porosity or the pore percentage, their increase will lead to a decrease in strength because total pore volume increases because of the increase in AEA content. Furthermore, the proportion of big and medium pores in the total pores increases, resulting in a decrease in the compactness of the AEACPB; therefore, the strength is reduced. 


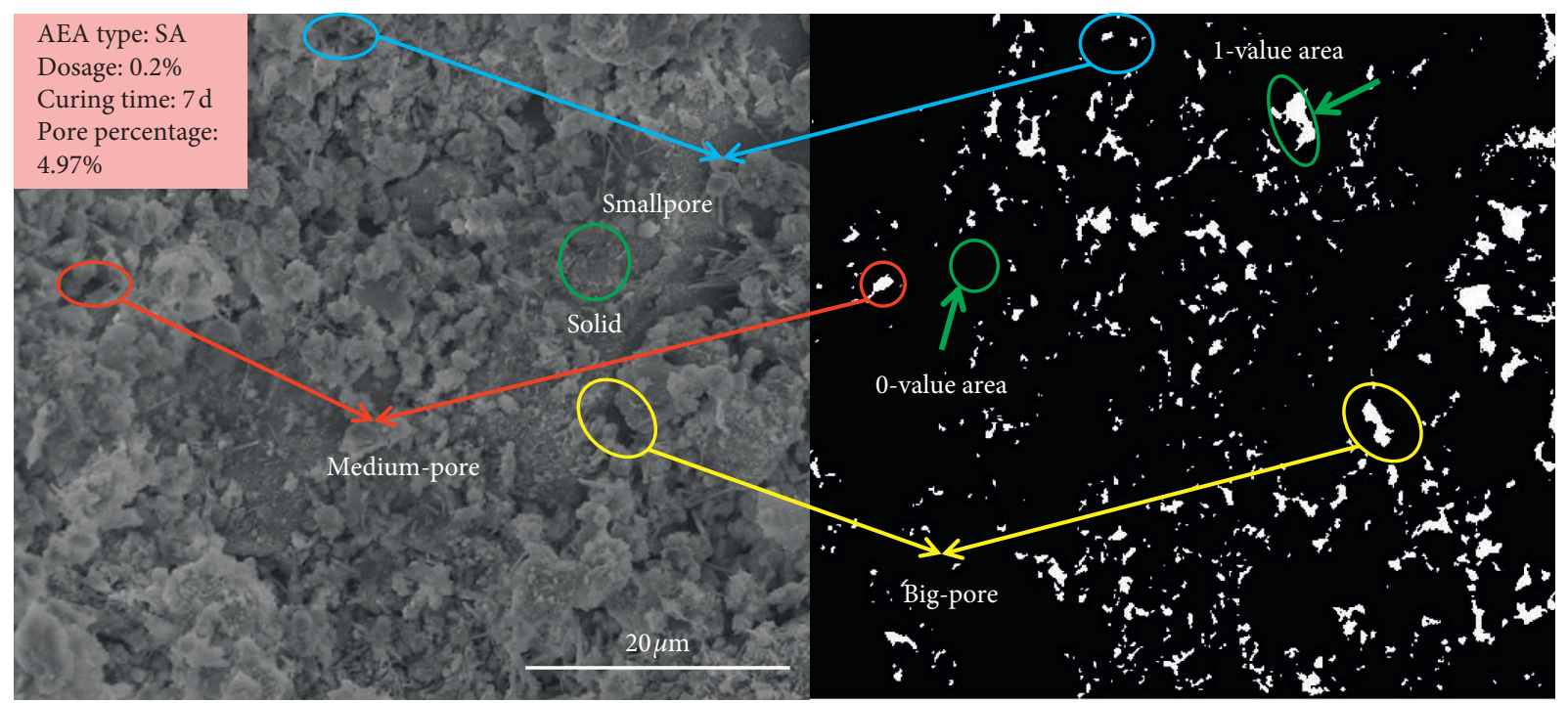

Figure 5: SEM and its binarization image of AEACPB.

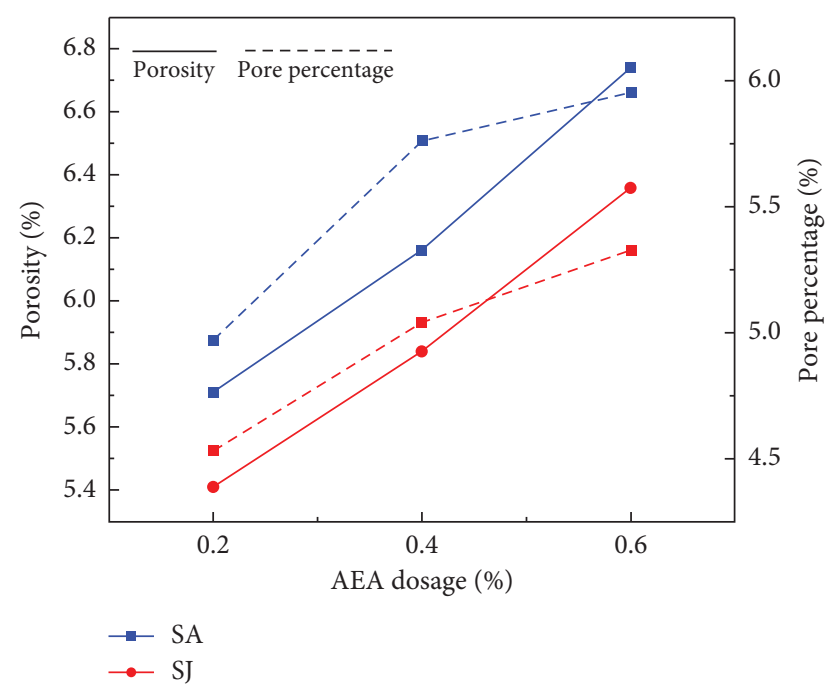

FIgURE 6: Changes of porosity and pore percentage with AEA content.

4.2. Relationship between Strength and $T_{2} S P A P$. Studies report that when the pore size is $>20 \mathrm{~nm}$, it is a harmful pore [22]. Note that there are three peaks on the $T_{2}$ spectrum, whereas the pore size is $>20 \mathrm{~nm}$. Here, we examined the effect of different pore size on strength. Figure 9 shows the relationships between the three $T_{2}$ spectrum peaks area percentage $\left(\mathrm{T}_{2} \mathrm{SPAP}\right)$ and the strength, respectively, where (a), (b), and (c) show the relationships between the first, second, and third peaks area percentage and the strength, respectively. Moreover, Figure 9 shows that there is a certain functional relationship between them, and the relationship is very good $\left(R^{2}>0.9\right)$. Furthermore, the relationships between them are as follows:

$$
\begin{aligned}
& y=0.63 x_{1}-56.38, \\
& y=-0.39 x_{2}+3.13
\end{aligned}
$$

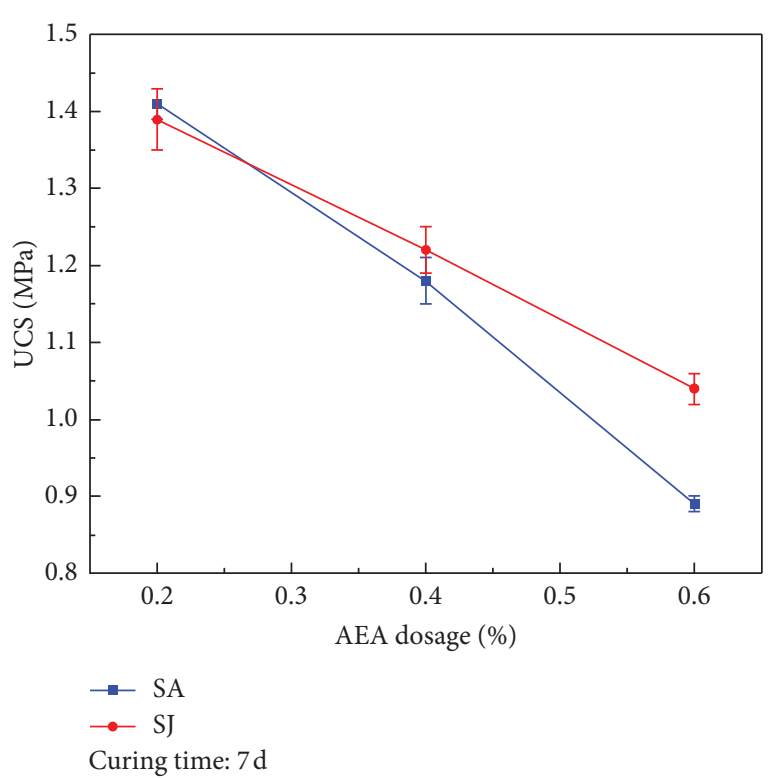

FIgURE 7: Variation characteristics of AEACPB strength with AEA content.

$$
y=-5.23 \times 10^{-20} e^{12.76 x_{3}}+1.38,
$$

where $y$ is strength (MPa) and $x$ is $T_{2} \operatorname{SPAP}(\%)$. Among them, $x_{1}$ is first peak, $x_{2}$ is second peak, and $x_{3}$ is third peak.

The above formulas show that the strength is linearly proportional related to the area percentage of the first peaks, linearly inversely related to the area percentage of the second peaks, and exponentially related to the area percentage of the third peaks. This demonstrates that when the total pores' volume of the AEACPB sample remains unchanged, the proportion of small pores is larger, and the strength increases linearly. However, when the proportion of medium pores is larger, the strength linearly decreases. Moreover, when the proportion of big pores increases, the strength 


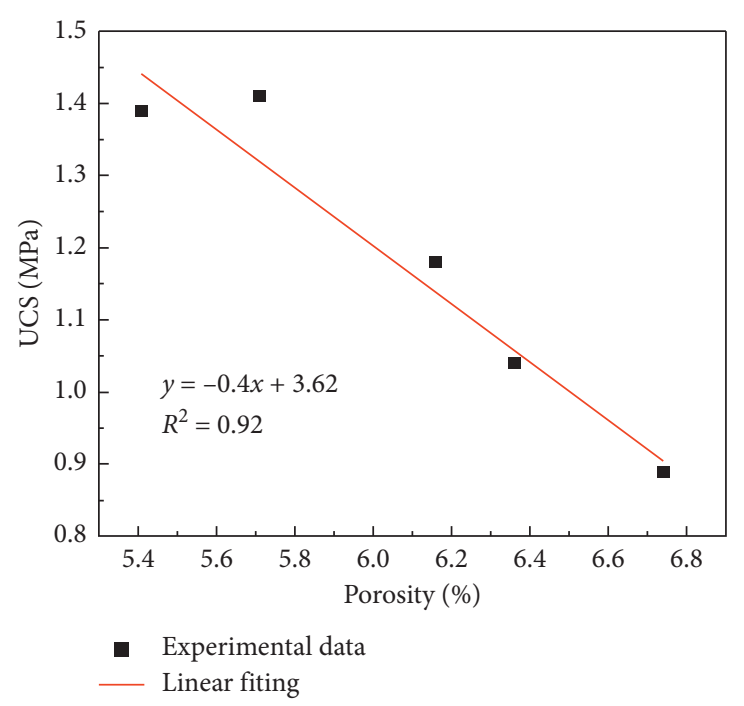

(a)

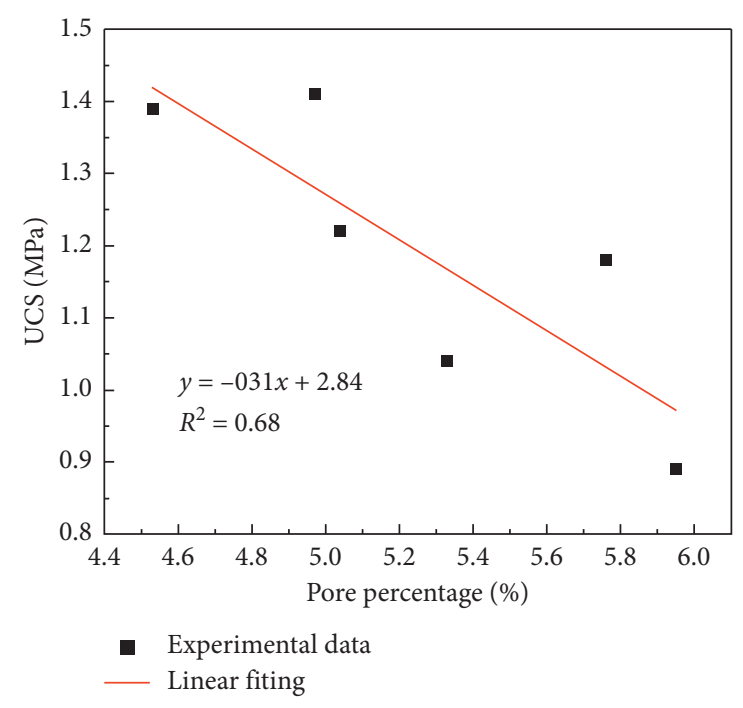

(b)

FIGURE 8: Relationship between strength and porosity and pore percentage.

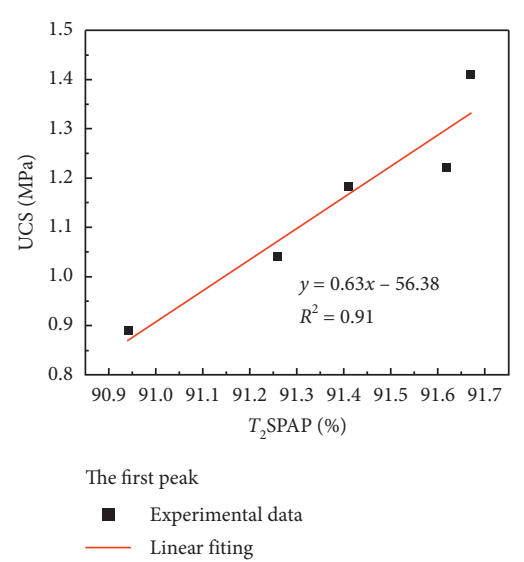

(a)

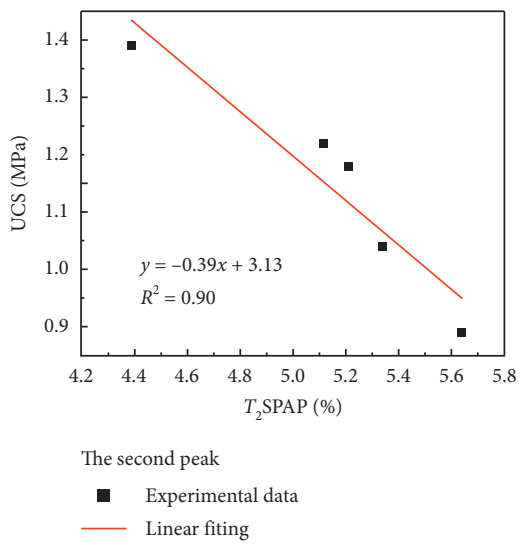

(b)

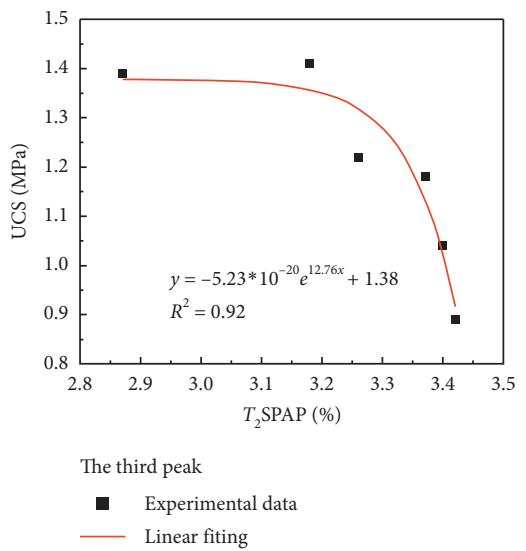

(c)

FIgURE 9: Relationship between strength and $T_{2}$ SPAP. (a) First peak; (b) second peak; and (c) third peak.

appears to exponentially decrease. This indicates that small pores have certain benefits on strength; however, medium pores have certain damage to strength and big pores have the greatest damage to strength. Therefore, the proportion of medium and big pores in the AEACPB should be minimized. Li et al. reported that more harmful pores having sizes of $>0.2 \mu \mathrm{m}$ have the greatest impact on strength reduction [27]. By comparing the slopes of equations (5) and (6), small pores have a greater and beneficial effect on strength. Figure 9 (c) shows that when the proportion of big pores reaches a certain amount, they have the greatest and harmful impact on strength; otherwise, the impact on strength is reduced. In these three types of pores, the amount of small pores should increase as much as possible, followed by the amount of medium pores. Furthermore, the amount of big pores should be avoided to exceed that fixed value as much as possible. If backfill needs the addition of AEA, note that a small amount of AEAs should be added, so that it is beneficial to the strength because it can effectively increase the proportion of small pores.

4.3. The Relationship between Fractal Dimension and UCS. As an important parameter of fractal geometry, fractal dimension can quantitatively describe the complexity of geometry. The combination of fractal theory and NMR can be used to study the pore characteristics in the AEACPB, so as to explain the change of strength from the complexity of pores. There is a certain functional relationship between the fractal dimension $D$ and $T_{2}$, and the relationship is as follows $[15,19]$ :

$$
l_{g}\left(S_{v}\right)=(3-D) l_{g}\left(T_{2}\right)+(D-3) l_{g}\left(T_{2 \max }\right)
$$

where $S_{v}$ is the percentage between pore cumulative volume with pore size less than $r$ and total pore volume and $T_{2 \max }$ is the $T_{2}$ value corresponding to the maximum pore size. 
TABLE 6: Fractal dimension of various pores.

\begin{tabular}{lcccccccc}
\hline \multirow{2}{*}{ Groups } & \multicolumn{3}{c}{ Small pore } & \multicolumn{3}{c}{ Medium pore } & \multicolumn{3}{c}{ Big pore } \\
& $k$ & $D_{s}$ & $R^{2}$ & $k$ & $D_{m}$ & $R^{2}$ & $k$ & $D_{b}$ \\
\hline SA1 & 2.599 & 0.401 & 0.88 & 0.034 & 2.966 & 0.98 & 0.018 & 2.982 \\
SA2 & 2.039 & 0.961 & 0.82 & 0.031 & 2.969 & 0.99 & 0.011 & 2.989 \\
SA3 & 1.707 & 1.293 & 0.81 & 0.03 & 2.97 & 0.97 & 0.012 & 2.988 \\
SJ1 & 2.584 & 0.416 & 0.88 & 0.032 & 2.968 & 0.98 & 0.016 & 2.984 \\
SJ2 & 2.227 & 0.773 & 0.87 & 0.037 & 2.963 & 0.98 & 0.017 & 2.983 \\
SJ3 & 1.621 & 1.379 & 0.86 & 0.031 & 2.969 & 0.97 & 0.015 & 2.985 \\
\hline
\end{tabular}

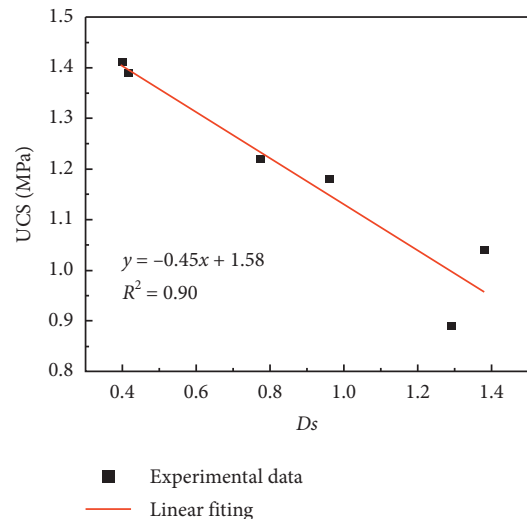

(a)

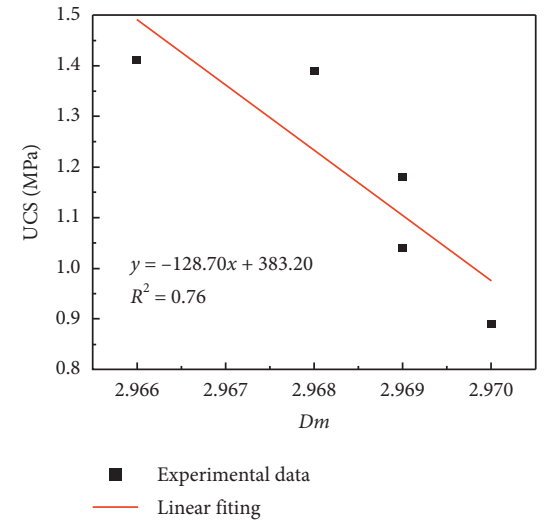

(b)

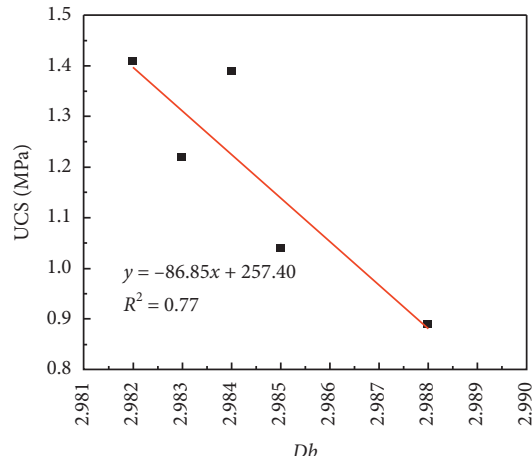

- Experimental data - Linear fiting

(c)

FIGURE 10: The relationship between UCS and the fractal dimension of various pores.

It can be found from equation (8) that the relationship between $\lg \left(S_{v}\right)$ and $\lg \left(T_{2}\right)$ is linear, and the sum of the slope $k$ and $D$ is 3 . The results shown in Table 6 can be obtained through the calculation of formula (8). By fitting the relationship between the fractal dimension and strength of various types of pores, we can get the result as shown in Figure 10. Among them, $D s, D m$, and $D b$ represent the fractal dimensions of small, medium, and big pores, respectively. It can be seen from Table 6 that $R^{2}$ of the small pores is all above 0.8 , indicating that the fractal property of the small pores is good. The fractal dimensions of big and medium pores are all 0.97 and above, indicating that the fractal properties of big and medium pores are very good. It can be seen from Figure 10 that $R^{2}$ of the relationship between the fractal dimension of small pores and strength is 0.9 , while that of medium pores and big pores is 0.76 and 0.77 , respectively, which indicates that the relationship between fractal dimension of small pores and strength is strong, while that of medium pores and big pores is weak. The functional relationship between them is as follows:

$$
\begin{aligned}
& y=-0.45 x_{1}+1.58, \\
& y=-128.70 x_{2}+383.20, \\
& y=-86.85 x_{3}+257.40,
\end{aligned}
$$

where $y$ represents UCS $(\mathrm{MPa})$, and $x$ represents the fractal dimension. Among them, $x_{1}$ is $D s, x_{2}$ is $D m$, and $x_{3}$ is $D b$.

It can be seen from Figure 10 that the strength decreases with the increase of fractal dimension. This is similar to the result obtained by Ding Ziwei and others in the study of the relationship between the pore fractal dimension and strength of sandstone, and Xie Chao and others also obtained similar results in the study of concrete [28, 29]. There are two reasons for this phenomenon, because the larger the fractal dimension, the more complex the pores. Firstly, the larger the fractal dimension is, the more pores with larger pore size will be, and the compactness of AEACPB will be decreased, which will cause great damage to the strength and reduce the strength. Studies have shown that the pore size becomes larger as the fractal dimension increases [28]. Second, the larger the fractal dimension, the more types of pore shapes. Irregular pores have a certain influence on the strength, and a large number of irregular pores reduce the strength.

\section{Conclusion}

The pore structure of the backfill has significant effect on strength. Note that AEA is added to regulate the pore structure of the backfill. Moreover, UCS, NMR, and SEM were used for the tests, and the relationships between pores' structure and backfill strength were quantitatively studied.

(1) For NMR analysis of AEACPB, there are three primary peaks in the $T_{2}$ spectrum, which can be divided into big, medium, and small pores as per the size of the pore structure. The pore size is primarily small pores (the largest area). As the content of the 
AEA increases, both the area of each peak and the total area are gradually increasing; however, the proportion of the first peak area is decreasing.

(2) The strength of the AEACPB is linearly negative correlation with porosity and pore percentage. Because the porosity and pore percentage increase, the strength of the AEACPB decreases.

(3) Pore structures of different scales have different action mechanisms on AEACPB strength. When the total pores in the AEACPB are constant, the higher the proportion of small pore, the strength linearly increases; the higher the proportion of medium pore, the strength linearly decreases; and the higher the proportion of big pore, the strength decreases exponentially. To improve the AEACPB strength, the external AEA should be adjusted as much as possible to effectively increase the amount of small pores, followed by the amount of medium pores, and avoid the amount of big pores.

(4) Through the analysis of fractal theory, all types of pores are fractal, and the fractal dimension has a linear negative correlation with the UCS.

\section{Data Availability}

The data used to support the findings of this study are available from the corresponding author upon request.

\section{Conflicts of Interest}

The authors declare that they have no conflicts of interest regarding the publication of this paper.

\section{Acknowledgments}

The authors gratefully acknowledge the support of the National Key Research and Development Program of China through Grant no. 2017YFC0602901 and the support of the National Natural Science Foundation of China (Grant no. 41672298). They are also thankful for the instructional support specialist Modern Analysis and Testing Central of Central South University.

\section{References}

[1] T.-Y. Qi, G.-R. Feng, Y.-R. Li, Y. Guo, J. Guo, and Y. Zhang, "Effects of fine gangue on strength, resistivity, and microscopic properties of cemented coal gangue backfill for coal mining," Shock and Vibration, vol. 2015, Article ID 752678, 11 pages, 2015.

[2] W. Xu, P. Cao, and M. Tian, "Strength development and microstructure evolution of cemented tailings backfill containing different binder types and contents," Miner, vol. 8, no. 4, pp. 1-15, 2018.

[3] Q. Zhang, Z. Wang, S. Rong et al., "Early strength characteristics and microcosmic influence mechanism analysis of cemented filling body of full tailings in deep mine," Nonferrous Metals Engineering, vol. 9, no. 6, pp. 97-104, 2019.
[4] W. Li and M. Fall, "Sulphate effect on the early age strength and self-desiccation of cemented paste backfill," Construction and Building Materials, vol. 106, pp. 296-304, 2016.

[5] H. Rong, M. Zhou, and H. Hou, "Pore structure evolution and its effect on strength development of sulfate-containing cemented paste backfill," Miner, vol. 7, no. 1, pp. 1-9, 2017.

[6] W. Xu, W. Pang, and M. Ding, "Experiment on evolution of microstructures and long-term strength model of cemented backfill mass," Journal of Central South University, vol. 46, no. 6, pp. 2333-2341, 2015.

[7] X. Li, B. Wand, J. You et al., "Study on mechanical properties and microstructure of the cemented tailings backfill," China Mining Magazinne, vol. 25, no. 6, pp. 169-172, 2016.

[8] W. Zhang, B. Li, H. Wang et al., "Analysis of pore structures and their relations with strength of hardened cement paste," Journal of Wuhan University of Technology-Materials Science and Engineering, vol. 20, no. 1, pp. 114-125, 2005.

[9] H. Zhao, Q. Xiao, D. Huang, and S. Zhang, "Influence of pore structure on compressive strength of cement mortar," The Scientific World Journal, vol. 2014, Article ID 247058, 12 pages, 2014.

[10] S. Jin, J. Zhang, and S. Han, "Fractal analysis of relation between strength and pore structure of hardened mortar," Construction and Building Materials, vol. 135, pp. 1-7, 2017.

[11] Y. Dai, P. Gao, H. Lin et al., "Effect of pore structure on the compressive strength of foamed concrete," New Building Materials, vol. 3, pp. 130-133, 2018.

[12] J. Huo, X. Cao, and X. Wang, "Influence of natural pumice concrete pore structure on its compressive strength," Journal of the Chinese Ceramic Society, vol. 39, no. 3, pp. 734-739, 2020.

[13] Y. Chang, "Study on the effect of different air entraining agents on the performance of concrete," Contemporary Chemical Industry, vol. 47, no. 10, pp. 2114-2117, 2018.

[14] J. H. Hu, Y. Kuang, T. Zhou, and F. Zhao, "Influence of air entraining agent on strength and microstructure properties of cemented paste backfill," IEEE Access, vol. 7, pp. 140899140907, 2019.

[15] J. Hu, F. Zhao, Y. Kuang, D. Yang, M. Zheng, and L. Zhao, "Microscopic characteristics of the action of an air entraining agent on cemented paste backfill pores," Alexandria Engineering Journal, vol. 59, no. 3, pp. 1583-1593, 2020.

[16] L. Jiang and Y. Guan, "Pore structure and its effect on strength of high-volume fly ash paste," Cement and Concrete Research, vol. 29, no. 4, pp. 631-633, 1999.

[17] C. Zou, G. Long, Y. Xie et al., "Evolution of multi-scale pore structure of concrete during steam-curing process," Microporous Mesoporous Mater, vol. 288, pp. 1-11, 2019.

[18] J. Hu, Q. Ren, Q. Jiang, R. Gao, L. Zhang, and Z. Luo, "Strength characteristics and the reaction mechanism of stone powder cement tailings backfill," Advances in Materials Science and Engineering, vol. 2018, Article ID 8651239, 14 pages, 2018.

[19] J.-H. Hu, Q.-F. Ren, D.-J. Yang et al., "Cross-scale characteristics of backfill material using NMR and Fractal Theory," Transactions of Nonferrous Metals Society of China, vol. 30, no. 5, pp. 1347-1363, 2020.

[20] L. Liu, Z. Fang, C. Qi, B. Zhang, L. Guo, and K.-I. Song, "Experimental investigation on the relationship between pore characteristics and unconfined compressive strength of cemented paste backfill," Construction and Building Materials, vol. 179, pp. 254-264, 2018.

[21] J. Hu, F. Zhao, Q. Ren et al., "Microscopic characterization and strength characteristics of cemented backfill under 
different humidity curing conditions," Royal Society Open Science, vol. 6, pp. 1-14, 2019.

[22] Z. Jiang, H. Deng, T. Liu, G. Tian, and L. Tang, "Study on microstructural evolution of marble under cyclic dynamic impact based on NMR," IEEE Access, IEEE Access, vol. 7, pp. 138043-138055, 2019.

[23] J. Li, H. Liu, K. Ai et al., "An NMR-based experimental study on the pore structure of the hydration process of mine filling slurry," Advances in Civil Engineering, vol. 2018, Article ID 4720356, 12 pages, 2018.

[24] Z. Wu and H. Lian, High-Performance Concrete, China Railway Press, Beijing, China, 1999.

[25] J. Hu, Q. Ren, X. Ding et al., "Trans-scale relationship analysis between the pore structure and macro parameters of backfill and slurry," Royal Society Open Science, vol. 6, no. 6, pp. 1-16, 2019.

[26] J. Hu, Q. Jiang, Q. Ren et al., "Cross scale correlation characteristics of pore structure and meso parameters of filling body," Transactions of Nonferrous Met. Metals Society of China, vol. 28, no. 10, pp. 2154-2163, 2018.

[27] W. Li, Z. Wang, L. Guo et al., "Correlation between early strength and pore structure of cemented tailing backfill sample," China Mining Magazinne, vol. 27, no. 10, pp. 143$147,2018$.

[28] C. Xie, Q. Wang, S. Li et al., "Relations of pore fractral dimension to pore structure and compressive strength of concrete under different water to binder ratio and curing condition," Journal of the Chinese Ceramic Society, vol. 34, no. 12, pp. 3695-3702, 2015.

[29] Z. Ding, X. Li, Q. Tang et al., "Study on correlation between fractal characteristics of pore distribution and strength of sandstone particles," Chinese Journal of Rock Mechanics and Engineering, vol. 39, pp. 1-10, 2020. 